\title{
Development of a Fortified Juice Beverage: Assessment of Iron Bioavailability Using an Established Caco-2 Cell Bioassay
}

\author{
Lisa Fleige ${ }^{1^{*}}$, Rachana Bhoite ${ }^{2}$, Ankita Marwaha ${ }^{3}$ and Raymond Glahn ${ }^{4}$ \\ ${ }^{1}$ Global R\&D Nutrition Sciences, PepsiCo, Inc., Chicago, IL, USA \\ ${ }^{2} R \& D$ Nutrition Sciences, PepsiCo India Holdings Pvt. Ltd \\ ${ }^{3} R \& D$ Nutrition Sciences, PepsiCo India Holdings Pvt. Ltd \\ ${ }^{4}$ Courtesy Associate Professor, Department of Food Science, Cornell University, USDA-ARS, Robert Holley Center for Agriculture and Health
}

Received: March 29, 2018; Accepted: May 11, 2018; Published: May 18, 2018

*Corresponding author: Lisa Fleige, Senior Principal Scientist, PepsiCo, Inc. 555 W. Monroe St. Chicago, IL 60661 USA, Tel: 312-821-2872; Fax No:312-821-1271, E-mail: Lisa.Fleige@pepsico.com

\begin{abstract}
Poor iron bioavailability is a contributing factor to the high prevalence of iron deficiency anemia in India, and food fortification with bioavailable iron is one strategy to help address the problem. Validated in vitro methods to measure iron (Fe) bioavailability are useful tools that can be leveraged to estimate the bioavailability of Fe in foods and beverages. In the present study, the objective was to determine if $15 \%$ of the Indian Recommended Dietary Allowance (RDA) of Fe as Micronized Iron Pyrophosphate (MIP) in $200 \mathrm{~mL}$ (i.e. $3.2 \mathrm{mg}$ Fe per $200 \mathrm{~mL}$ ) of a fortified mixed Fruit and Vegetable Juice Beverage (FJB) provides bioavailable Fe. To test this objective, various amounts of MIP were added to the FJB with 3 different levels of ascorbic acid (i.e. vitamin C; a known enhancer of Fe bioavailability and an essential nutrient). Vitamin A (retinyl acetate) at a level of 90 $\mu \mathrm{g} / 200 \mathrm{~mL}$ was also added to all samples, except for some controls. The results demonstrated that 15\% RDA of Fe (as MIP) in a $200 \mathrm{~mL}$ serving of juice fortified with equimolar ascorbic acid resulted in good Fe bioavailability for this form of Fe. Overall, the study demonstrated that a FJB product could be developed to be an excellent source of dietary Fe.
\end{abstract}

Keywords: Fortified juice beverage, iron bioavailability, in vitro, Caco-2 cell bioassay, iron fortification

Abbreviations: $\mathrm{RDA}=$ recommended dietary allowance; $\mathrm{FJB}=$ fortified juice beverage; MIP = micronized iron pyrophosphate, $\mathrm{NaFeEDTA}$ = sodium iron ethylenediaminetetracetic acid; $\mathrm{FWB}=$ fortified water beverage; $\mathrm{FBC}=$ fortified breakfast cereal; $\mathrm{AA}=$ ascorbic acid; vitamin $\mathrm{C}$

\section{Introduction}

Iron Deficiency Anemia (IDA) is the most prevalent micronutrient deficiency, affecting up to 2 billion people globally including more than half of all women of reproductive age (15-49 years) in India $[1,2]$. Despite three decades of national supplementation programs iron deficiency continues to be a grim public health problem in India existing across income quintiles, age groups and gender (24 percent men) [3]. Multiple factors including inadequate dietary intake and low bioavailability of iron have contributed to the high incidence of IDA. The Indian government considers food fortification an effective means to address micronutrient deficiencies. The Food Safety Standards Authority of India published draft standards for food fortification in 2016 and supports voluntary fortification of wheat flour and rice via state-wide initiatives [4]. These initiatives are well aligned with the global recommendations of the World Health Assembly (WHA). However, WHA also highlights that "a diet containing adequate amounts of bioavailable iron should underpin all efforts for prevention and control of anemia" [5]. Selecting and testing an appropriate food vehicle and fortificant for iron bioavailability are important considerations to help improve nutritional status and reduce the prevalence of IDA.

Iron (Fe) can be present in the human diet in either the heme or non-heme forms. Heme Fe is from hemoglobin, most commonly found in red meats, and is seldom used as a food fortificant due to cost, processing issues, shelf life, color and cultural issues. Non-heme iron is present in plant foods that contain inhibitors of Fe absorption such as phytic acid, oxalic acid and certain polyphenols. Heme Fe is considered to be highly available relative to non-heme Fe as it is not affected by these inhibitors. In general, it is believed that individuals who consume diets with moderate amounts of heme Fe seldom experience Fe deficiency; whereas those consuming diets lacking in heme Fe are more at risk for $\mathrm{Fe}$ deficiency [6].The high prevalence of iron deficiency in India may be partially attributed to iron-poor vegetarian diets that include consumption of green leafy vegetables, fruits and pulses that do not significantly impact anemia status [7]. Non-heme forms of Fe are the most common form of fortificants used in fortifying food products. In recent years, many forms of non-heme Fe have been developed and utilized in a broad range of food and beverage products and these fortificants exhibit considerable variance in bioavailability depending on the nature of the food product. In 
general, reduced or elemental $\mathrm{Fe}(\mathrm{Fe} 0)$ tends to have the lowest bioavailability, whereas forms such as Ferrous Sulphate (FeSO4), Sodium Iron EDTA (NaFeEDTA), ferric pyrophosphate, and encapsulated forms are similar in bioavailability with occasional differences due to unique interactions with the food matrix [8]. Indeed the choice of Fe form is often determined more by organoleptic interactions with a given food, while simultaneously balancing the nutritional label, focusing on content with little concern as to bioavailability [9].

The nutritional quality (i.e. bioavailability) of Fe in any given food product or meals determined by the interactive effects of the intrinsic form(s) of the Fe in the food matrix, the form of the fortified $\mathrm{Fe}$, the amount of the fortified $\mathrm{Fe}$, and the overall solubility of the Fe as it interacts with components of the food or meal both before and during digestion. For many food products, Fe bioavailability is often not assessed as the producer is not aware of the potential benefits of doing so, or is simply willing to assume that the formulation will provide significant absorbable Fe based on content alone.

The primary objective of the present study was to utilize an established Caco- 2 cell bioassay for Fe bioavailability to assure that a Fortified Mixed Fruit And Vegetable Juice Beverage (FJB) containing Micronized Iron Pyrophosphate (MIP) (15\% Indian RDA), Ascorbic Acid (AA) (15\% Indian RDA) and vitamin A (15\% Indian RDA as retinyl acetate), also a nutrient of public health concern in India, per $200 \mathrm{ml}$ serving would deliver substantial absorbable Fe [8]. In addition, the study was designed to

1) Determine the proper level of ascorbic acid needed to maintain the Fe bioavailability in FJB;

2) Explore if additional $\mathrm{Fe}$ would increase the amount of bioavailable Fe in FJB;

3) Compare the bioavailability of MIP to sodium iron EDTA (NaFeEDTA) in FJB;

4) Compare Fe bioavailability from the FJB when consumed in the presence of spinach.

Spinach is often mistakenly assumed to be a rich source of Fe by some Indian consumers; therefore, we measured Fe content and assessed Fe bioavailability from spinach alone and in combination with the FJB to demonstrate this nutrition myth. The study also assessed Fe bioavailability in two other commercial Fe fortified products, a Fe Fortified Water Beverage (FWB) and a $\mathrm{Fe}$ Fortified Breakfast Cereal (FBC), available in the Indian market.

\section{Materials and Methods}

\section{Sample Source and Preparation}

The unfortified juice was prepared at PepsiCo India and shipped frozen to Cornell University. Samples were prepared immediately prior to the experiment, at Cornell University as per the amounts listed in Table 1. Both Fe and AA stock solutions were prepared fresh immediately prior to the experiment. MIP and NaFeEDTA were supplied by DSM Nutritional Products,
Mumbai, India. Vitamin A in the form of retinyl acetate was supplied by Sigma-Aldrich (CAS Number 127-47-9). Ascorbic acid was supplied by Sigma (CAS Number 50-81-7).

Aside from the juice formulations, a FWB product (Sample 17) and FBC (Sample 18) were tested. Fresh baby spinach was purchased from a local grocery store in the United States and $2 \mathrm{~g}$ (wet weight) was included in samples 19-22. Fe content for these foods is shown in Table 1.

\section{Iron Content Analysis}

Fe content of all samples was conducted via inductively coupled plasma emission spectroscopy (ICP-ES). To do so, $0.5 \mathrm{ml}$ liquid or $0.5 \mathrm{~g}$ solid samples were dried down and then treated with $3.0 \mathrm{~mL}$ of 60:40 HNO3 and HClO4 mixture into a Pyrex glass tube and left for overnight to destroy organic matter. The mixture was then heated to $120^{\circ} \mathrm{C}$ for two hours and $0.25 \mathrm{~mL}$ of $40 \mu \mathrm{g} / \mathrm{g}$ yttrium added as an internal standard to compensate for any drift during the subsequent inductively coupled plasma atomic emission spectrometer (ICP-AES) analysis. The temperature of the heating block was then raised to $145^{\circ} \mathrm{C}$ for $2 \mathrm{~h}$. Then, the temperature of the heating block was raised to $190^{\circ} \mathrm{C}$ for ten minutes and then turned off. The cooled samples in the tubes were then diluted to $20 \mathrm{~mL}$, vortexed and transferred into auto sample tubes to analyze via ICP-AES. The model of the ICP used was a Thermo iCAP 6500 series (Thermo Jarrell Ash Corp., Franklin, MA, USA).

\section{Fe Bioavailability Analysis}

An established Caco-2 cell bioassay was utilized to provide measurement of relative Fe bioavailability between samples [10]. This model uses Caco-2 cell ferritin formation as a marker of $\mathrm{Fe}$ uptake. Caco-2 cells are an established human intestinal cell line that is commonly used to study intestinal absorption of nutrients. In these cells, the intracellular Fe storage protein known as ferritin forms in direct proportion to cell Fe uptake. Measurement of this protein provides a sensitive and accurate measurement of cell Fe uptake, negating the needs for more expensive methods such as isotopic labelling.

When conducting a bioassay such as the one used in this study, it is important to utilize quality controls to ensure that a valid run of the bioassay was performed. As in all runs of this model system in the Glahn lab, the quality controls are known as baseline, $\mathrm{Fe}, \mathrm{Fe}$ + AA, and a dehulled red lentil. The baseline sample is merely all of the solutions used in the bioassay, including the digestive enzymes. For the Fe control, $56 \mu \mathrm{g}$ of FeCl3 $(1 \mu \mathrm{mole})$ is added. For the $\mathrm{Fe}+\mathrm{AA}, 56 \mu \mathrm{g} \mathrm{FeCl} 3$ and $3.50 \mathrm{mg} \mathrm{AA}(20 \mu$ moles$)$ are added. The lentil sample contains $55 \mu \mathrm{g} \mathrm{Fe} / \mathrm{g}$ sample dry weight. As per the experimental samples, 3 independent replicates were run for quality controls. In the present study, the Caco-2 cell ferritin values (mean \pm standard deviation) for these controls were: Baseline, $3.3 \pm 0.4 ; \mathrm{Fe}, 27.0 \pm 4.8$; Fe $+\mathrm{AA}, 501.1 \pm 21.7$; lentil, 13.0 \pm 1.1. Units were $\mathrm{ng}$ ferritin per mg cell protein. Baseline values are expected to be low, i.e. $\leq 5$, which indicates that minimal contamination of Fe was present in the system. The Fe sample 
Development of a Fortified Juice Beverage: Assessment of Iron Bioavailability Using an Established Caco-2 Cell Bioassay

Table 1: Description of the samples used for the experiment

\begin{tabular}{|c|c|c|c|c|c|c|c|}
\hline Sample & $\begin{array}{c}\% \text { RDA } \\
\text { Fe }\end{array}$ & $\begin{array}{c}\% \text { RDA } \\
\text { AA }\end{array}$ & $\begin{array}{c}\text { Molar Ratio } \\
\text { AA:Fe }\end{array}$ & Sample Description ${ }^{1}$ & Fe (mg) & AA (mg) & Vit. A ( $\mu \mathrm{g})$ \\
\hline 1 & 0 & 0.0 & NA & Juice (unfortified) & 0 & 0 & 0 \\
\hline 2 & 15 & 0.0 & NA & FJB (Fe as MIP) & 3.2 & 0 & 0 \\
\hline 3 & 15 & 15.0 & $0.60: 1$ & $\mathrm{FJB}(\mathrm{Fe}$ as MIP) + AA & 3.2 & 6 & 0 \\
\hline 4 & 15 & 15.0 & $0.60: 1$ & $\begin{array}{c}\text { FJB (Fe as MIP })+\mathrm{AA}+ \\
\text { Vit. A }\end{array}$ & 3.2 & 6 & 90 \\
\hline 5 & 17 & 32.5 & $0.87: 1$ & $\begin{array}{c}\text { FJB (Fe as MIP) + AA } \\
\text { + Vit. A }\end{array}$ & 3.6 & 13 & 90 \\
\hline 6 & 20 & 35.0 & $1.05: 1$ & $\begin{array}{c}\text { FJB (Fe as MIP) + AA } \\
+ \text { Vit. A }\end{array}$ & 4.2 & 14 & 90 \\
\hline 7 & 15 & 37.5 & $1.5: 1$ & $\begin{array}{c}\text { FJB (Fe as MIP) + AA } \\
+ \text { Vit. A }\end{array}$ & 3.2 & 15 & 90 \\
\hline 8 & 20 & $65.0 \%$ & $1.96: 1$ & $\begin{array}{c}\mathrm{FJB}(\mathrm{Fe} \text { as MIP })+\mathrm{AA} \\
+ \text { Vit. A }\end{array}$ & 4.2 & 26 & 90 \\
\hline 9 & 15 & 50.0 & $2: 01$ & $\begin{array}{c}\mathrm{FJB}(\mathrm{Fe} \text { as MIP })+\mathrm{AA} \\
+ \text { Vit. A }\end{array}$ & 3.2 & 20 & 90 \\
\hline 10 & 15 & 65.0 & $2.6: 1$ & $\begin{array}{c}\mathrm{FJB}(\mathrm{Fe} \text { as MIP })+\mathrm{AA} \\
+ \text { Vit. A }\end{array}$ & 3.2 & 26 & 90 \\
\hline 11 & 15 & 82.5 & $3.3: 1$ & $\begin{array}{c}\mathrm{FJB}(\mathrm{Fe} \text { as MIP })+\mathrm{AA} \\
+ \text { Vit. A }\end{array}$ & 3.2 & 33 & 90 \\
\hline 12 & 15 & 100.0 & $3.96: 1$ & $\begin{array}{c}\mathrm{FJB} \text { (Fe as MIP) + AA } \\
\text { + Vit. A }\end{array}$ & 3.2 & 40 & 90 \\
\hline 13 & 15 & 125.0 & $4.95: 1$ & $\begin{array}{c}\text { FJB (Fe as MIP) + AA } \\
\text { + Vit. A }\end{array}$ & 3.2 & 50 & 90 \\
\hline 14 & 15 & 0 & NA & $\begin{array}{c}\text { FJB (Fe as NaFeEDTA) } \\
+ \text { Vit. A }\end{array}$ & 3.2 & 0 & 90 \\
\hline 15 & 15 & 15.0 & $0.60: 1$ & $\begin{array}{c}\text { FJB (Fe as NaFeEDTA) } \\
+ \text { AA + Vit. A }\end{array}$ & 3.2 & 6 & 90 \\
\hline 16 & 15 & 25.0 & 1:01 & $\begin{array}{c}\text { FJB (Fe as NaFeEDTA) } \\
+ \text { AA + Vit. A }\end{array}$ & 3.2 & 10 & 90 \\
\hline 17 & 16 & NA & NA & FWB & 3.36 & 0 & 0 \\
\hline 18 & 25 & 25.0 & NA & $\mathrm{FBC}$ & 5.25 & 10 & 30 \\
\hline 19 & 0 & NA & NA & $\begin{array}{l}\text { Spinach alone (serving } \\
\text { size }=100 \mathrm{~g} \text { wet basis) }\end{array}$ & NA & NA & $\mathrm{NA}$ \\
\hline 20 & 15 & 50.0 & $2: 01$ & $\begin{array}{c}\text { FJB (Fe as MIP) + Vit. } \\
\text { A + AA+ spinach }\end{array}$ & 3.2 & 20 & 90 \\
\hline 21 & 15 & 37.5 & NA & $\begin{array}{l}\text { FJB (Fe as MIP) + Vit. } \\
\text { A + AA + spinach }\end{array}$ & 3.2 & 15 & 90 \\
\hline 22 & 0 & 50.0 & NA & $\begin{array}{l}\text { Juice (no iron) + Vit. A } \\
+ \text { A + spinach }\end{array}$ & NA & 20 & 90 \\
\hline
\end{tabular}

${ }^{1} \mathrm{NA}=$ not applicable

Fortified Juice Beverage (FJB)

Fortified Water Beverage (FWB)

Fortified Breakfast Cereal (FBC)

Ascorbic acid (AA) and Vitamin A (Vit. A)

Citation: Fleige L, Rachana B, Ankita M, Raymond G (2018) Development of a Fortified Juice Beverage: Assessment of Iron 
ferritin value was within the range of $23-90 \mathrm{ng} / \mathrm{mg}$ cell protein, and as expected the AA should enhance the ferritin formation demonstrating the responsiveness of the cells to highly available $\mathrm{Fe}+2$ created by the 20:1 molar ratio of AA added to the $\mathrm{FeCl} 3$. The value of $\mathrm{Fe}+\mathrm{AA}$ control is usually in the range of $250-550 \mathrm{ng}$ ferritin/mg cell protein. The lentil sample is used to represent a solid food sample, and is normally in the range of 6-20 ng ferritin/ mg cell protein. It is important to note that this system, like most bioassays, has a range of responsiveness that can vary from one run to the next. However, as is also true of most bioassays, the positive and negative controls exhibit responsiveness in parallel thus making the bioassay useful as it defines relative differences. This bioassay can accommodate up to 22 samples plus the quality controls; thus, all samples can be directly compared in this study as they were analyzed on the same run of the bioassay.

Caco-2 cell growth is also important to monitor when using this bioassay. Healthy Caco-2 cell monolayers, grown under the culture conditions of this model, should yield 2.5-3.0 mg cell protein per $9.6 \mathrm{~cm} 2$ well at 14 days post seeding. In the present study, the Caco- 2 protein levels were $2.6 \pm 0.1 \mathrm{mg}$ cell protein per well, indicating consistent and healthy growth across the multiwell plates.

\section{Statistical Analysis}

Data were analyzed using the software package GraphPad Prism (GraphPad Software, San Diego, CA). Data were analyzed using analysis of variance incorporating normalization of variance, if needed, and Tukey's post test to determine significant differences $(\mathrm{P}<0.05)$ between groups. Unless noted otherwise values are expressed as mean \pm 1 standard deviation; $\mathrm{n}=3$ independent replications.

\section{Results}

The FJB used in the present study contained only 1.1 $\mu \mathrm{g}$ intrinsic Fe per ml; thus, essentially all of the $\mathrm{Fe}$ in the experimental sample came from the fortified Fe. The unfortified juice exhibited no difference from the baseline control, thus indicating no significant intrinsic bioavailable Fe in the juice.

The Fe content of the spinach was $10.3 \mu \mathrm{g}$ Fe per g wet weight. The FWB contained $17.7 \mu \mathrm{g}$ Fe per ml equivalent to $3.54 \mathrm{mg}$ per $200 \mathrm{~mL}$ serving.

The FBC contained 227.6 $\mu \mathrm{g}$ Fe per g dry weight, equivalent to $6.8 \mathrm{mg}$ Fe per serving size of $30 \mathrm{~g}$.

As only 3 levels of Fe were used in the study (i.e. 15, 17 and

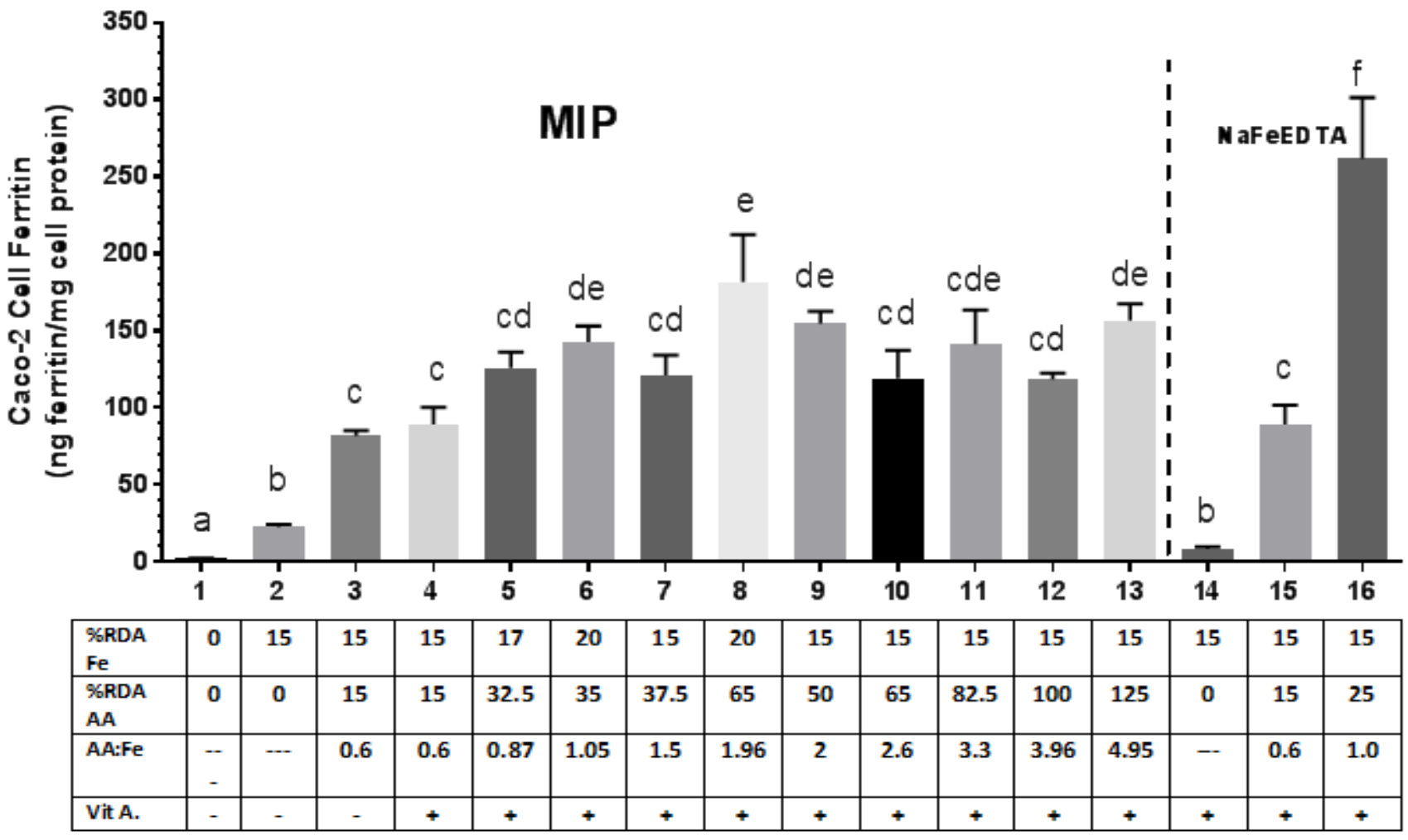

Figure 1: Relative Fe bioavailability, expressed as Caco-2 cell ferritin formation, of unfortified juice [1], fortified juice beverage (FJB) with micronized iron pyrophosphate (MIP) [2-13], FJB with sodium iron EDTA (NaFeEDTA) [14-16] as shown in table below the $\mathrm{x}$ axis. Values represent mean+standard deviation of 3 replicates. Columns with no letters in common are significantly different $(P<0.05)$ 
$20 \%$ Indian RDA), only a small effect of Fe content on higher Fe uptake was possible in the present study. Such effect was likely part of the increase in ferritin formation observed across samples 3-8 Figure 1. However, across this same range of samples the AA levels were also increasing.

AA is known to enhance Fe uptake, and this effect is first observed in comparison of samples 2 versus 3 Figure 1 . As additional amounts of AA were added in samples $4-13$, a maximal effect of added AA becomes evident as no additional increase in ferritin formation occurs.

In the FJB matrix, bioavailability of the MIP was similar to
NaFeEDTA when Fe and AA content was the same, as evident in comparison of samples 3 versus 15 Figure 1 . However, increased AA with NaFeEDTA resulted in the highest ferritin formation observed with the FJB formulations (sample 16, Figure 1).

The Caco- 2 cell ferritin values from the FWB and FBC were $35.3 \pm 1.9$ and $169.7 \pm 10.7$, respectively Figure 2 .

Fresh spinach has relatively low $\mathrm{Fe}$ content and low $\mathrm{Fe}$ bioavailability, exhibiting a ferritin value of $2.0 \pm 0.1$, statistically equal to baseline Figure 2 . The presence of spinach decreased Fe uptake when added to the juice (samples 7 and 9 versus 20 and 21).

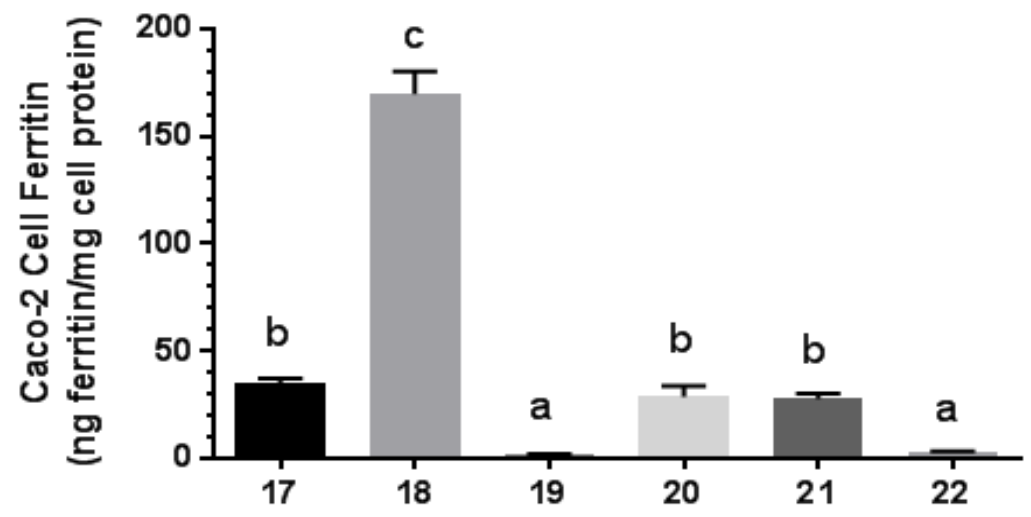

\begin{tabular}{|c|c|c|c|c|c|c|}
\hline \%RDA Fe & 16 & 25 & 0 & 15 & 15 & 0 \\
\hline $\begin{array}{c}\text { \%RDA } \\
\text { AA }\end{array}$ & 0 & 25 & NA & 50 & 37.5 & 50 \\
\hline AA:Fe & NA & $0.6: 1$ & NA & $2: 1$ & 1.5 & NA \\
\hline Vit A. & NA & NA & NA & + & + & + \\
\hline
\end{tabular}

Figure 2: Relative Fe bioavailability, expressed as Caco-2 cell ferritin formation, of the fortified water beverage (FWB) [17], the fortified breakfast cereal (FBC) [18], fresh spinach [19], spinach plus the fortified juice beverage (FJB) with varying AA and Fe (in the form of MIP) [20,21], and spinach plus juice (with no iron) [22] as shown in table below the $\mathrm{x}$ axis. Values represent mean +standard deviation of 3 replicates. Columns with no letters in common are significantly different $(P<0.05)$.

\section{Discussion}

The present study exemplifies how this validated Caco2 bioassay can be used to optimize a FJB to improve $\mathrm{Fe}$ bioavailability [11].

The results demonstrate that the 15\% RDA of Fe generates a relatively strong Caco- 2 cell ferritin formation. This indicates that the FJB should be an good source of Fe fortification. If consumed with other foods high in inhibitory factors, such as spinach, then the bioavailability of the Fe could likely decrease Figure 2. Components in the spinach matrix, such as oxalic acid may have contributed to the decrease. It is also possible that the simple addition of solid plant matter contributed to the effect. Fe can bind quite easily to solid background material and become unavailable for uptake.

To fully understand the Fe bioavailability results, one must be familiar with the mechanism of action of AA on non-heme Fe. AA reduces $\mathrm{Fe}+3$ (ferric) to $\mathrm{Fe}+2$ (ferrous) without complexing the $\mathrm{Fe} . \mathrm{Fe}+2$ is soluble at near neutral $\mathrm{pH}$ and thus is free to interact with the Fe uptake transporter, and thus be taken up by the intestinal epithelial cell, the first step in Fe absorption, and the primary factor in Fe bioavailability. For this Fe uptake to occur, AA must be present in the reduced form in relatively equal or slightly higher molar ratio than the Fe. AA also counteracts the presence of inhibitory compounds present in the food matrix or diet, such phytic acid, citric acid or polyphenols which can complex Fe and compete with the uptake transporter for the available $\mathrm{Fe}$ depending on their concentration and the AA levels. It is the net sum of the above effects that ultimately controls Fe bioavailability.

Given that the food product matrix is liquid, the $\mathrm{Fe}$ bioavailability is primarily a function of the amount of added Fe, the solubility of the form of the Fe in the FJB matrix (ie. MIP vs. 
NaFeEDTA), the relative amount of AA, and the relative amounts of other factors in the FJB such as citric acid, polyphenols or other unknowns. Although not analyzed in this study, phytic acid is unlikely to be present in any significant amount in the FJB. Polyphenols were not measured in this study, but given the results, inhibitory polyphenols do not appear to be present in any significant amounts $[12,13]$

Overall fortifying with $15 \%$ RDA of Fe and with a molar AA:Fe ratio equal to 1 is good option for the following reasons:

Although adding 20\% RDA of Fe resulted in more absorbable iron, this amount may be infeasible as it could affect the organoleptic quality of the juice. Also, with more Fe, additional AA would need to be added to maintain the benefit over the shelf life of the product, and this may be a challenge in India given the regulatory restrictions which limit addition of AA to $\leq 40 \mathrm{mg}$ per serving [15].

Shelf life is an important consideration with addition of AA as factors such as time, temperature, $\mathrm{pH}$ and oxygen can play a role in oxidation of $\mathrm{AA}$ and thus reduce levels of $\mathrm{AA}$ and hence Fe bioavailability [16]. Thus, sufficient overage of AA must be added to maintain the molar AA:Fe level at a level greater than or equal to 1 . In the present study the AA was added to the samples immediately before the start of the experiment; hence there was little time for AA degradation to occur. Of note, the intrinsic AA level in the unfortified juice sample was not measured but the FJB is not expected to deliver any significant amount of intrinsic AA.

The NaFeEDTA form of Fe yielded a similar Fe bioavailability relative to the MIP form when the AA:Fe ratio was 0.6; but when the AA:Fe ratio was increased to 1.0 the Fe uptake from the NaFeEDTA formulation was significantly higher, even more than when additional Fe and AA was added to the MIP formulation (Figure 1; samples 8 versus 16). Using this form of Fe may be infeasible due to cost and/or potential formulation issues. One should also consider that the MIP form adds substantial bioavailable Fe to the FJB, thus it is a strong nutritional product for delivering more Fe based on the fortificant levels explored in the present study.

Finally, in regards to the FBC and FWB, given the cereal is solid and the FJB and FWB are liquids, relative comparisons should only be useful based on the amount of Fe present in the in vitro digestion. For the FBC, the Fe bioavailability was quite high, relatively equal to the highest values observed with the FJB formulations. However, the FBC also had Fe added at a concentration of $25 \%$ RDA, substantially more than the FJB formulations. The lower Fe bioavailability from the FWB may be partially attributed to it not containing any AA.

In conclusion, the present study clearly defines a target formulation of $15 \%$ RDA Fe with a molar AA:Fe ratio $\geq 1$ that enhances Fe bioavailability, and suggests the FJB maybe an appropriate vehicle to deliver bioavailable $\mathrm{Fe}$ to the Indian population where prevalence of Fe deficiency is a significant public health concern.

\section{Conflict of Interest and Funding Disclosure}

This study was sponsored by PepsiCo, Inc. R\&D Nutrition Sciences. Lisa Fleige, Ankita Marwaha and Rachana Bhoite are full-time employees of PepsiCo, Inc., which manufactures juice products under the brand name Tropicana. Raymond Glahn is a USDA employee, and Courtesy faculty of Cornell University. The views expressed in this report are those of the authors and do not necessarily represent the position or policy of PepsiCo, Inc, USDA or Cornell University.

\section{Ethical Approval: NA}

\section{Clinical Trial registration: NA}

\section{References}

1. Allen L, de Benoist B, Dary O, Hurrell R. Guidelines on food fortification with micronutrients. World Health Organization. 2009;ISBN 9241594012.

2. International Institute for Population Sciences (IIPS) and ICF. 2017. National Family Health Survey (NFHS-4). 2015-16;India, Mumbai: IIPS.

3. Ministry of Health and Welfare. Government of India National Iron + Initiative. Guidelines for control of iron deficiency anaemia. 2013.

4. Food Safety and Standards Authority of India. Journey of Food Fortification. 2016;13

5. World Health Organization. World Health Assembly Global Nutrition Targets 2025: Anaemia policy brief.2014;4.

6. Hurrell R, Egli I. Iron bioavailability and dietary reference values. Am J Clin Nutr. 2010;91(5):1461S-1467S. doi: 10.3945/ ajcn.2010.28674F

7. Rammohan A, Awofeso N, Robitaille M. Addressing female irondeficiency anaemia in India: is vegetarianism the major obstacle? International Scholarly Research Notices. 2012;7-8.

8. Wortley G, Leusner S, Good C, Gugger E, Glahn R. Iron availability of a fortified processed wheat cereal: a comparison of fourteen iron forms using an in vitro digestion/human colonic adenocarcinoma (Caco-2) cell model. Br J Nutr. 2005;93(1):65-71.

9. Uauy R, HertrampfE, Reddy M. Iron fortification of foods: overcoming technical and practical barriers J Nutr. 2002;132(4):849S-52S. doi: 10.1093/jn/132.4.849S

10. Glahn R, Lee O, Yeung A, Goldman M, Miller D. Caco-2 cell ferritin formation predicts non-radiolabeled food iron availability in an in vitro digestion/Caco-2 cell culture model. J Nutr. 1998;128(9):15551561.

11. Tako E, Bar H, Glahn R. The combined application of the Caco2 cell bioassay coupled with in vivo (Gallus gallus) feeding trial represents an effective approach to predicting Fe bioavailability in humans. Nutrients. 2016;8(11):732. doi:10.3390/nu8110732

12. Hart JJ, Tako E, Glahn R. Characterization of polyphenol effects on inhibition and promotion of iron uptake by Caco-2 cells. J Agric Food Chem. 2017;65(16):3285-3294. doi: 10.1021/acs.jafc.6b05755

13. Boato F, Wortley GM, Liu R, Glahn R. Red grape juice inhibits iron availability: application of an in vitro digestion/Caco-2 cell model. J Agric Food Chem. 2002;50(23):6935-6938. 
14. Glahn R, Tako E, Cichy KA, Wiesinger J. The cotyledon cell wall of the common bean (phaseolus vulgaris) resists digestion in the upper intestine and thus may limit iron bioavailability. Food and Function. 2002;7:3193.

15.The Gazette of India. Part 3; section 4. Ministry of Health and family welfare. Food Safety and Standards Authority of India, New Delhi:2016
16. García-Torres R, Ponagandla N R, Rouseff R L, Goodrich-Schneider R M, Reyes-De-Corcuera J I. Effects of dissolved oxygen in Fruit Juices and Methods of Removal. Comprehensive Reviews in Food Science and Food Safety. 2009;8:409-423. 